UCLA/05/TEP/21

\title{
Astrophysical bounds on supersymmetric dark-matter Q-balls
}

\author{
Alexander Kusenko ${ }^{1}$, Lee C. Loveridge ${ }^{1}$, and \\ Mikhail Shaposhnikov ${ }^{2}$ \\ ${ }^{1}$ Department of Physics and Astronomy, UCLA, Los Angeles, CA 90095-1547 \\ ${ }^{2}$ Institute of Theoretical Physics, Ecole Polytechnique Fédérale de Lausanne, \\ SB/ITP/LPPC, BSP 720, CH-1015, Lausanne, Switzerland
}

\begin{abstract}
.
Stable baryonic Q-balls, which appear in supersymmetric extensions of the Standard Model, could form at the end of cosmological inflation from fragmentation of the Affleck - Dine condensate. We reconsider astrophysical constraints on such Q-balls as dark matter candidates. Baryonic Q-balls interact with matter by absorbing the baryon number and, effectively, leading to a rapid baryon number non-conservation. We have recently shown that this process can occur at a much faster rate than that used in previous calculations. As a consequence, stability of neutron stars imposes a stringent constraint on the types of Q-balls that can be dark matter. Only the Q-balls that correspond to baryonic flat directions lifted by baryon-number violating operators are allowed as dark-matter candidates.
\end{abstract}




\section{Introduction}

Supersymmetry (SUSY) provides two types of dark matter candidates: the lightest supersymmetric particle (e.g., neutralino or gravitino), and stable Q-balls. SUSY Qballs are nontopological solitons that carry baryon number [1, 2, 3]. They are either stable or have lifetimes in excess of the age of the universe in theories with gaugemediated SUSY breaking [4. At the end of inflation, large SUSY Q-balls can form via fragmentation of the Affleck-Dine condensate [5, 6, 17].

SUSY Q-balls can interact with matter fermions. A quark scattering off a baryonic Q-ball can convert into an antiquark, while increasing the baryon number of the Q-ball. Hence, Q-balls catalyze baryon number non-conservation in the sector of ordinary, nonsupersymmetric particles. The effective B-nonconservation catalyzed by Q-balls was considered in Ref. [8] in connection with astrophysical limits on Q-ball dark matter. However, it was recently shown [9] that the rate of this process is considerably higher than that used in Ref. [8]. In this paper we reconsider astrophysical limits on dark matter Q-balls and show that a wide variety of them are ruled out because they would destabilize neutron stars on cosmologically short time scales.

\section{Interactions of SUSY Q-balls with matter}

In a previous paper [9] we reanalyzed the interaction of Q-balls with ordinary matter. We found that quarks falling on a Q-ball are reflected as antiquarks with a probability of order one, practically independent of the parameters of the theory. In other words, Q-balls convert the matter into antimatter on their surface (or antimatter into matter, if placed in an anti-matter environment). Baryon number is conserved during this process: after reflection of an antinucleon, the baryonic charge of the Q-ball increases by 2 units.

Having described the interaction of SUSY Q-balls with nucleons, we now reexamine some bounds on dark-matter Q-balls. The laboratory limits do not change significantly from the earlier estimates [8]. This is because nucleons reflected from the Q-ball surface as antinucleons have a very small mean free path in the surrounding matter. They annihilate quickly, mainly into pions. The energy release is somewhat higher than it was thought to be [8], but this is an order one effect, which does not make a difference for most bounds.

However, the analysis of astrophysical bounds, in particular those from Q-balls captured in the neutron stars, must be done differently. The rate of baryon number absorption by a Q-ball was underestimated in Ref. [10] by several orders of magnitude. One can expect a dramatic change in the astrophysical bounds because of this. In fact, such rapid absorption of nucleons requires that we reexamine the inner fluid dynamics of the neutron star. Although the dynamics of nuclear matter is difficult to describe, we understand it well enough to know that certain types of Q-balls would consume a neutron star in a few thousand years. This is far less than the lower bound lifetime of neutron stars and suggests that such Q-balls must not exist in large amounts in the 
universe.

We will also address another important issue overlooked in the earlier work. Nonrenormalizable operators alter the flat directions in the potential and can modify the shapes of solutions for SUSY Q-balls. We will consider interactions of Q-balls of different types.

\section{SUSY Q-balls and the flat directions}

Stable Q-balls develop a VEV along some flat direction. This flat direction can be parameterized by a scalar field $\varphi$. The VEVs of squarks and sleptons are proportional to $\varphi$. In theories with gauge-mediated breaking of supersymmetry, the effective potential along this flat direction is nearly constant, $V(\varphi) \propto \Lambda^{4}$, up to some $\varphi_{\max }$ which depends on the type of the flat direction $\dagger$. There exists a classical spherically symmetric solution of the field equations, a non-topological soliton, in which

$$
\varphi=\phi_{0} f(r) e^{i \omega t}
$$

where $f(r) \simeq \sin (\omega r) /(\omega r)$ for $r \leq R=\pi / \omega$ and zero for $r>R$. It carries the global charge $Q$. If $\phi_{0}<\varphi_{\max }$, different parameters depend on $Q$ as follows:

$$
\phi_{0} \sim \Lambda Q^{1 / 4}, \quad M \sim \Lambda Q^{3 / 4}, \omega \sim \Lambda Q^{-1 / 4} .
$$

We shall call the non-topological solitons of this type flat direction (FD) Q-balls.

Flat directions are in general lifted by higher dimension non-renormalizable operators [11] and the effective potential starts to grow at $\varphi>\varphi_{\max }$. Thus, when the charge $Q$ exceeds some critical value $Q_{c}$, the properties of Q-balls change. The details of this analysis will appear elsewhere [12], here we reproduce the essentials only.

Generally, the lifting terms can be written in the form

$$
V^{n}(\phi)_{\text {lifting }} \approx \lambda_{n} M^{4}\left(\frac{\phi}{M}\right)^{n-1+m}\left(\frac{\phi^{*}}{M}\right)^{n-1-m},
$$

where the smallest possible $n$ and corresponding to it $m$ are some integers that are determined by the structure of the flat direction and can be found from Tables 4 and 5 of ref. [11. We will assume that $\lambda_{n}$ is of the order one and that $M$ is the grand unification scale $\sim 10^{16} \mathrm{GeV}$ and that $\Lambda \sim 10^{3} \mathrm{GeV}$.

The relations (21) are valid only if the lifting potential is small compared with $\Lambda^{4}$. So, the Q-ball properties must be re-analyzed when their charge reaches the critical value $Q_{c}$ determined from the condition $V^{n}(|\phi|)_{\text {lifting }} \simeq \Lambda^{4}$, and equal to

$$
Q_{c} \simeq \lambda_{n}^{-\frac{2}{n-1}}\left(\frac{M}{\Lambda}\right)^{\frac{4 n-12}{n-1}}
$$

It can be seen from [11] that most flat directions are lifted by monomials of dimension $n=4$. At this low suppression, only $Q$-balls with small charge $Q<Q_{c} \simeq 10^{17}$ can retain their $\propto Q^{3 / 4}$ energy dependence despite the lifting. On the other end, all flat $\dagger$ We omit possible log factors, because they are not essential for the discussion. 
directions are lifted by monomials of dimension $n=7$, which corresponds to a critical charge $Q_{c} \simeq 10^{34}$.

The fate of Q-balls with charges larger than $Q_{c}$ depends crucially on the value of the integer $m$ in (3). For $m \neq 0$ the lifting potential not only breaks the degeneracy of the flat direction, but introduces an explicit breaking of the global U(1) symmetry responsible for Q-ball existence. For these flat directions non-renormalizable operators induce strong baryon number non-conservation, which leads to fast Q-ball decay. As the value of $Q_{c}$ happens to be much smaller than the baryon number of a star, no astrophysical limits on this type of Q-balls can be derived. These Q-balls absorb only a small fraction of the baryon material of a star and disappear afterwords. In notations of ref. [11] Q-balls related to flat directions $Q L e, Q L d$, Lude, QLde, QLud, QLude are of this type.

If, on the contrary, $m=0$, the lifting term does not break the global U(1) symmetry and the Q-ball charge can grow beyond $Q_{c}$, but the dependence of the parameters of the Q-ball on Q is modified. Now, with the lifting terms incorporated, the quantity $V(\varphi) /|\varphi|^{2}$ does have a minimum at non-zero finite value of $\varphi$, and the analysis of [1] is applicable. Thus,

$$
\phi_{0} \sim \varphi_{\max }, \quad M \sim \mu Q, \quad \omega \sim \mu, \quad R \sim\left(\mu Q / \Lambda^{4}\right)^{1 / 3},
$$

where $\mu^{2} \sim V\left(\varphi_{\max }\right) / \varphi_{\max }^{2}$. Changing $n$ from $n=4$ to $n=7$ the frequency $\omega$ ranges from $10 \mathrm{MeV}$ to $10^{-3} \mathrm{MeV}$ and the VEV $\phi_{0}$ from $10^{7}$ to $10^{11} \mathrm{GeV}$. In notations of ref. [1] Q-balls related to $u d$, ue, $Q L$, ude flat directions are of this type. In the following we will be interested in these Q-balls only.

Though in general the critical charge $Q_{c}$ depends on the structure of the flat direction and on parameters of the underlying unified model [12], the exact values of the critical charge and of $\omega$ are of little importance for us. What is essential is that (i) $Q_{c}$ is much smaller than the baryon charge of the neutron stars $Q_{\mathrm{ns}} \sim 10^{57}$; (ii) $\omega$ is much smaller than the nucleon mass and smaller than the chemical potential for the baryon number in the center of a neutron star; and (iii) that $\phi_{0} \gg \omega$ for dark matter Q-balls. These types of non-topological solitons shall be dubbed CD (curved directions) Q-balls.

Very large Q-balls can become black holes. For FD Q-balls this happens when the global charge reaches

$$
Q_{\mathrm{bh}} \simeq 8 \cdot 10^{61}\left(\frac{T e V}{\Lambda}\right)^{4},
$$

and for CD Q-balls the corresponding number is

$$
Q_{\mathrm{bh}} \simeq 2 \cdot 10^{53}\left(\frac{\mathrm{TeV}}{\Lambda}\right)^{2}\left(\frac{\mathrm{MeV}}{\omega}\right) .
$$

While for FD Q-balls it exceeds the typical baryonic charge of a star, for CD Q-balls it is less than $Q_{\mathrm{ns}}$, which is important for discussion below.

A summary of the Q-ball properties can be found in Table I. 


\begin{tabular}{|c|c|c|}
\hline & FD & CD \\
\hline$\varphi$ & $\frac{1}{\sqrt{2}} \Lambda Q^{1 / 4}$ & $\varphi_{\max }$ \\
\hline$\omega$ & $\pi \sqrt{2} \Lambda Q^{-1 / 4}$ & $\Lambda^{2} \varphi_{\max }^{-1}=\pi \sqrt{2} \Lambda Q_{c}^{-1 / 4}=\omega_{c}$ \\
\hline$M$ & $4 \pi \frac{\sqrt{2}}{3} \Lambda Q^{3 / 4}$ & $\omega Q$ \\
\hline$R$ & $\frac{1}{\sqrt{2} \Lambda} Q^{1 / 4}$ & $\left(\frac{3}{8 \pi} \frac{1}{\Lambda^{2} \varphi_{\max }} Q\right)^{1 / 3}=\left(\frac{3}{2}\right)^{1 / 3}\left(Q / Q_{c}\right)^{1 / 12} R_{F D}$ \\
\hline
\end{tabular}

Table 1. Summary of properties of Q-balls of different types

\section{Astrophysical limits}

We now ask whether such a large processing rate of baryonic matter by the relic SUSY Qballs could affect some astrophysical observations which could be used to either detect dark-matter Q-balls or rule them out. Q-balls are extremely dense and heavy and, therefore, they do not stop in most astrophysical objects, such as stars or planets [10]. They would stop, however, in both neutron stars and white dwarfs. Furthermore, simple estimates suggest that most neutron stars and white dwarfs are infected by Q-balls within a short period of time [10]. Neutron stars and white dwarfs are, therefore, the most reasonable places to look for Q-ball signals.

A Q-ball captured by a neutron star or a white dwarf begins consuming baryonic matter. At some pooint, the neutron star mass can go below its stability limit, which is about $0.2 M_{\odot}[13$. Then the neutron star explodes. This happens when a CD Q-ball inside becomes a black hole. If the time scale for this process is less than the known ages of some neutron stars and, then such Q-balls must not exist. Spindown rates of some pulsars is as low as $(\dot{P} / P) \sim(0.3-3) \times 10^{-10} \mathrm{yr}^{-1}$, which corresponds to the age in billions of years. Some pulsars are also known to be (at least) as old as 10 Gyr based on the cooling ages of their white dwarf companions [14]. One must, therefore, rule out all Q-balls that would destroy neutrons stars in less than $10^{10}$ years.

As we have already mentioned, the estimate of the neutron star lifetime made in Ref. [10] was based on an incorrect rate of the baryon number absorption by nontopological solitons. In this section we will revise and correct this analysis. We shall also incorporate two other effects that were not essential for the analysis in Ref. [10] because of the assumed small rate of the baryon number transfer from the neutron matter to Q-balls. The first effect is related to the fact that the rate of baryon number transformation on the surface of Q-balls is large, and, therefore, there is a large pressure from the radiated pions. In this case the rate of absorption is determined by the fluid dynamics and relative pressures of neutrons and pions rather than by the probability of matter-antimatter transformation on the Q-ball surface. The second effect is that the charge-energy relations are changing after the Q-ball reaches the critical charge discussed in Section 2, see eq. (5). Unfortunately, relatively little is known about the internal structure of neutron stars and white dwarfs. Therefore, we will present two simplified models to get an idea of the timescale for neutron star and white dwarf consumption. 


\subsection{Unitarity bound}

Let us present a lower bound on the lifetime of a neutron star infested with Q-balls. Let us assume that the baryon number density near the Q-ball surface is given by the nuclear density $n_{0} \sim 4 \cdot 10^{-3} \mathrm{GeV}^{3}$ at the center of the neutron star without a Q-ball 13. Then the absorption rate simply coincides with the number of baryons falling on the Q-ball,

$$
\frac{d Q}{d t} \simeq \pi r_{Q}{ }^{2} n_{0} v,
$$

where $v$ is the typical nucleon velocity, taken to be of the order of the speed of light in what follows.

Let us now assume that the nuclear density in the center of the star does not change with time (this is a conservative assumption because the central density decreases when the baryon number of the neutron star is consumed by a Q-ball). Then the lifetime of a star populated by FD Q-balls is of the order of

$$
t \sim \frac{\Lambda^{2}}{n_{0}}\left(Q_{\mathrm{ns}}\right)^{1 / 2} \sim 10^{5} \text { years } .
$$

For the CD Q-balls (a more realistic case, as the FD Q-balls are transformed into CD Q-balls after their charge increases past $Q_{c}$ ) the lifetime is much smaller,

$$
t \sim \frac{\left(\omega_{c} \varphi_{\max }^{2}\right)^{2 / 3}}{n_{0}}\left(Q_{\mathrm{bh}}\right)^{1 / 3} \sim O(1) \text { day } .
$$

In contrast, a white dwarf is about $10^{6}$ times less dense than a neutron star, so the time scale for a white dwarf to be consumed is about $10^{11}$ years for a FD Q-ball and about $10^{3}$ years for a CD Q-ball.

All but one of these estimates are considerably smaller than the typical ages of neutron stars or white dwarfs. We will see, however, that the baryon number absorption is mainly determined by the transport properties of quarks and nucleons in the interior of the neutron stars and that the speed of baryon number consumption by Q-balls is in fact considerably smaller. Nevertheless, it happens to be large enough to completely exclude Q-balls (with $m=0$ in eq. (31)) as candidates for dark matter.

\subsection{Hydrodynamic Considerations}

It is clear that the unitarity limit is unreasonable. However, it is not clear what the appropriate transport mechanism inside the neutron star will be. We know that near the Q-ball neutrons will be consumed and turned into pions (either directly at the Qball surface, or in an annihilation). We also know that a neutron star is held stable by the interplay of gravity pulling inward and the tremendous degeneracy pressure of the neutrons pressing outward. If the change in neutron density results in a pressure change, then the transport will be dominated by pressure driven hydrodynamic flows. If instead, the pressure remains constant, then the transport will be dominated by either convection or diffusion. Therefore, we must first determine whether or not the pressure can remain constant near the Q-ball. 
There are two simple ways to estimate the pressure near the center of the star. Macroscopically, we can determine the required pressure to maintain the star in hydrostatic equilibrium. For a constant density the pressure is

$$
P=\frac{3}{8 \pi} \frac{M^{2}}{m_{p l}^{2}} \frac{1}{R^{4}} .
$$

Using $M \approx 10^{57} \mathrm{GeV}$, and $R \approx 10 \mathrm{~km} \approx 5 \times 10^{19} \mathrm{GeV}^{-1}$ yields $2 \times 10^{-4} \mathrm{GeV}^{4} \approx(.12 \mathrm{Gev})^{4}$.

In the second approximation we use the pressure from a degenerate non-relativistic fermi gas.

$$
\frac{\left(6 \pi^{2}\right)^{1 / 3}}{5 m} n^{5 / 3} .
$$

Using the density of the star as $10^{15} \mathrm{~g} / \mathrm{cm}^{3}$ we find that $P \approx 10^{-4} \mathrm{GeV}^{4} \approx(.1 \mathrm{GeV})^{4}$.

While both approximations are highly simplified, they give the same order of magnitude for the pressure which we assume is reliable. As the Q-ball consumes baryon number, the degeneracy of neutrons will no longer be able to maintain this high pressure. The pressure will then have to be maintained either thermally, or by pions before they are able to decay into other particles. We will see shortly that neither option is viable.

4.2.1. Thermal Pressure. The thermal pressure from light particles is

$$
P \approx g \frac{T^{4}}{\pi^{2}}
$$

where $g$ is the number of light degrees of freedom. In our case these are 2 photons, 4 electrons, 2 neutrinos and possibly 3 pions (depending on the exact temperature). This suggests that we will have an extrememly high temperature of about $100 \mathrm{MeV}$.

Such high temperatures can not be maintained because neutrinos carry the energy away faster than the Q-ball can produce it by absorbing neutrons. Therefore we know that the pressure near the Q-ball can not be maintained by thermal equilibrium.

4.2.2. Pion Pressure. While the pressure can not be maintained by thermal effects, we may hope that it is maintained by the extra pions produced before they are able to decay. Because the pions interact on strong length scales, but decay over electromagnetic and weak time scales, there is a significant time when they behave much like thermal conserved particles.

We will therefore assume that near the Q-ball, we have a mixture of neutrons and pions, each with an average kinetic energy of about .1 GeV, and that the pressure of either component is simply the number density times the kinetic energy. The pions will continuously change between species, but since the average kinetic energy is lower than their mass, no new pions will be produced. We shall also assume that once a pion decays, it no longer plays a significant role in the pressure of the system. This is because photons, neutrinos, and electrons all have much smaller cross sections than neutrons and pions, and they will immediately leave the area near the Q-ball. Any 
such particles entering the Q-ball region will have the much lower energies typical of the colder outside region.

In this situation, the pions will cluster near the Q-ball where they are produced, but they will wander away from the Q-ball essentially due to random walk diffusion. However, the distance they reach is limited by their lifetime $\tau$. Thus, they will reach an average distance from the Q-ball of about $\sqrt{3 \lambda \tau}$ We will model their density profile as a falling Gaussian with this decay length. A one dimensional model is sufficient because the decay length is much less than the radius of the Q-ball.

$$
n_{\pi}(x)=n_{p i}(0) \exp \left(-\frac{x^{2}}{3 \lambda \tau}\right) .
$$

Then the total rate of pion loss is, approximately,

$$
\begin{aligned}
\frac{d N_{\pi}}{d t} & =\frac{1}{3 \tau} 4 \pi r_{Q}{ }^{2} \int_{0}^{\infty} n_{p i}(0) \exp \left(-\frac{x^{2}}{\lambda \tau}\right) d x \\
& =2 \pi^{3 / 2} \sqrt{\frac{\lambda}{3 \tau}} n_{\pi}(0) r_{Q}{ }^{2} .
\end{aligned}
$$

To maintain pressure, $n_{\pi}(0)$ needs to be about the same as the density of neutrons far from the Q-ball, but still near the center of the star. That is $n_{0} \approx .1 \mathrm{GeV}^{3}$. We will also take $\lambda \approx n_{0}^{-1 / 3}$. That is we are letting the mean free path be about the spacing between particles. Finally $\tau \approx 10^{8} \mathrm{GeV}^{-1}$. (This is the lifetime of a neutral pion. We assume that the decay rate is dominated by the electro-magnetic decays of neutral pions.) From this we find

$$
\frac{d N_{\pi}}{d t}=10^{-16} Q^{2 / 3} \mathrm{GeV} .
$$

This is the rate at which pions are lost to decays. (If we require them to be lost through weak decays, the number changes to about $10^{-20}$. This is less of a change than we might expect because the lifetime only enters as $\tau^{-1 / 2}$ not as $\tau^{-1}$. In essence, the pions are decaying slower, but there are more of them. However, for such a situation to happen we must justify a lack of neutral pions. Given the frequent strong interactions this seems unlikely.) Now, one neutron can only supply about 4-5 pions, so the rate of baryon consumption required to maintain pressure near the Q-ball is no more than one order of magnitude below this. That means

$$
\begin{aligned}
& \frac{d N_{n}}{d t} \approx 10^{-17} Q^{2 / 3} \mathrm{GeV}, \\
& t \approx 3 \times 10^{17} Q_{B H}^{1 / 3} \mathrm{GeV}^{-1} \approx 1500 \text { years } .
\end{aligned}
$$

This is clearly much longer than the unitarity limit, but still well below the known lifetimes of neutron stars. Because they are smaller, grow more slowly, and must consume a larger number of neutrons, the lifetime of a FD Q-ball is much longer, about $10^{10}$ years.

The neutron star lifetimes are summarized in Table IV. 


\begin{tabular}{|c|c|c|}
\hline & FD Q-balls & CD Q-balls \\
\hline$t$ & $10^{10}$ years & 1500 years \\
\hline
\end{tabular}

Table 2. Neutron star lifetimes for Q-balls of different types.

Of course in reality, the neutron density near the Q-ball will be decreased slightly due to their rapid consumption. However, as we have shown this will result in a drop in pressure which will cause more neutrons to flow to the site. If the density dropped by several orders of magnitude, this would greatly increase the lifetime of the star. However, the pressure is so great that a slight (order 1 or less) drop in neutron density near the Q-ball creates a pressure gradient that is sufficiently large to replenish the consumed neutrons. Such a drop would not greatly change the lifetime of the neutron star.

The predicted lifetime of CD Q-balls is so small that we can clearly rule out their existence. If they existed there would be no neutron stars older than a few thousand years. FD Q-balls could still survive the astrophysics bound. However, as mentioned earlier, large FD Q-balls are unlikely to exist since all the flat directions are lifted for VEVs much smaller than those for which the Q-ball reaches the size of a neutron star.

However, this limit applies only to Q-balls with $m=0$, which conserve baryon number at all values of $Q$. Q-balls for which $m \neq 0$ do not grow beyond a certain size because the baryon number non-conserving interactions in their interior prevent $Q$ from growing beyond the critical value $Q_{c}$. Such Q-balls remain small, and, therefore, they do not consume a neutron star on cosmologically interesting time scales.

We note in passing that the corresponding analysis for the white dwarfs would be more complicated because the main pressure supporting a white dwarf against gravity comes from the Fermi pressure of degenerate electrons. This pressure is coupled only electromagnetically to the strong dynamics of nuclei and Q-balls. The surface temperature of the white dwarfs could be used to set a limit on the amount of heat generated inside by the Q-ball [15]. There are many 10 Gyr old white dwarfs that have cooled to very low temperatures and have luminosities as low as

$$
L_{\text {wd }}=3 \times 10^{-5} L_{\odot}=7 \times 10^{28} \mathrm{erg} / \mathrm{s} .
$$

However, we do not need to consider white dwarfs in detail because, for $m=0$, Qballs are already ruled out by the stability of the neutron stars. For $m \neq 0$, the rate of energy release from consumption of nuclear matter is much lower than the value in equation (19).

\section{Conclusions}

We have revised the bounds on the relic dark-matter Q-balls based on the existence of neutron stars and their stability on the time scale of billions of years. Assuming SUSY Q-balls have a sufficient abundance to be the dark matter, relic SUSY Q-balls 
are captured by a neutron star shortly after its formation. The Q-ball consumes ambient neutrons and adds to the baryon number of its condensate, while dissipating the excess energy in neutrinos and photons. Meanwhile, the neutron star mass decreases. For most SUSY Q-balls, this process is too rapid, and it can destabilize a neutron star on time scales much shorter than a billion years. Hence, such Q-balls are ruled out. The only SUSY Q-balls that survive this bound and remain dark-matter candidates are those that have their flat directions lifted by the baryon number violating operators. Such Q-balls cannot grow beyond a certain size, which turns out to be too small for a rapid consumption of nuclear matter.

Acknowledgments. The authors thank E.S. Phinney and J. Morris for valuable comments. A.K. and L.L. were supported in part by the US Department of Energy grant DE-FG03-91ER40662 and by NASA grants ATP02-0000-0151 and ATP03-00000057. The work of M.S. was supported in part by the Swiss Science Foundation. We thank Peter Tinyakov for helpful discussions.

\section{References}

[1] G. Rosen, J. Math. Phys. 9, 996 (1968) ibid. 9, 999 (1968); R. Friedberg, T. D. Lee, A. Sirlin, Phys. Rev. D 13, 2739 (1976); S. Coleman, Nucl. Phys. B 262, 263 (1985); A. Kusenko, Phys. Lett. B 404, 285 (1997).

[2] T. D. Lee and Y. Pang, Phys. Rept. 221, 251 (1992).

[3] A. Kusenko, Phys. Lett. B 405, 108 (1997).

[4] G. Dvali, A. Kusenko, M. Shaposhnikov, Phys. Lett. B 417, 99 (1998).

[5] I. Affleck, M. Dine, Nucl. Phys. B 249, 361 (1985); M. Dine, L. Randall, S. Thomas, Phys. Rev. Lett. 75, 398 (1995); Nucl. Phys. B 458, 291 (1996); R. Allahverdi, B. A. Campbell and J. R. Ellis, Nucl. Phys. B 579, 355 (2000); A. Anisimov and M. Dine, Nucl. Phys. B 619, 729 (2001); A. Anisimov, Phys. Atom. Nucl. 67, 640 (2004) [Yad. Fiz. 67, 660 (2004)]; M. Kawasaki, K. Konya and F. Takahashi, Phys. Lett. B 619, 233 (2005).

[6] M. Dine and A. Kusenko, Rev. Mod. Phys. 76, 1 (2004).

[7] K. Enqvist and A. Mazumdar, Phys. Rept. 380, 99 (2003).

[8] A. Kusenko, V. Kuzmin, M. Shaposhnikov, P. G. Tinyakov, Phys. Rev. Lett. 80, 3185 (1998).

[9] A. Kusenko, L. Loveridge and M. Shaposhnikov, arXiv:hep-ph/0405044.

[10] A. Kusenko, M. Shaposhnikov, P. G. Tinyakov, I. I. Tkachev, Phys. Lett. B 423, 104 (1998).

[11] T. Gherghetta, C. F. Kolda and S. P. Martin, Nucl. Phys. B 468, 37 (1996).

[12] M. Shaposhnikov and R. Tabbash, in preparation.

[13] Shapiro and Teukolsky, Black holes, white dwarfs, and neutron stars, John Wiley \& Sons, New York, 1983.

[14] B.M.S. Hansen, E.S. Phinney, Mon.Not.Roy.Astron.Soc. 294, 557 (1998); ibid., 294, 569 (1998).

[15] E.S. Phinney, private communication. 